\title{
Coma and Conciousness
}

\author{
Sibhi Ganapathy ${ }^{*}$, Swaroop Gopal ${ }^{2}$, T.V Ramakrishna ${ }^{3}$ \\ ${ }^{1}$ Consultant, ${ }^{2}$ Director Neurosciences Senior Consultant, ${ }^{3}$ Associate Consultant, Dept. of Neurosurgery, Manipal Hospital Whitefield, \\ Bangalore, Karnataka, Sakra World Hospital, Bengaluru, Karnataka, India
}

*Corresponding Author: Sibhi Ganapathy

Email: sibhig@yahoo.co.uk

\begin{abstract}
The concepts of consciousness, coma and wakefulness have fascinated man from the ages. The explanation and thereby, understanding of different states of altered consciousness vary from mystic, spiritual to anatomic and scientific. The advent of experimental neurology and anatomy gradually expanded the scientific horizons of man allowing observations and measurements to be made and interpreted.
\end{abstract}

Keywords: Coma, Conciousness.

The concepts of consciousness, coma and wakefulness have fascinated man from the ages. The explanation and thereby, understanding of different states of altered consciousness vary from mystic, spiritual to anatomic and scientific. The advent of experimental neurology and anatomy gradually expanded the scientific horizons of man allowing observations and measurements to be made and interpreted.

An epoch braking understanding occurred with the discovery of the Ascending Reticular Activating System (ARAS), by an Austrian Nobleman Baron Constantin von Economo who demonstrated the seminal influence of the ARAS in wakefulness in animal models. Further research discovered the presence of Orechxin as well as Seratonin influencing the neurotransmitter release in the ARAS as well as adjoining sections of the Hypothalamus.
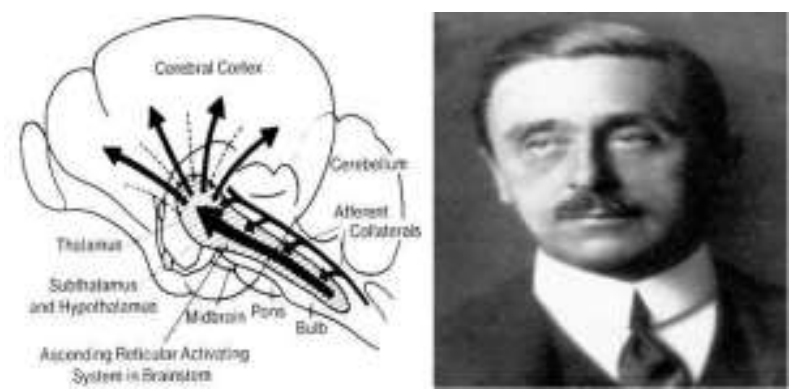

Fig. 1-2: Baron CONSTANTIN von ECONOMO and the aras

Plum and Posner through trial and error attempted to bring order into the chaos that was the study on consciousness by finally defining conditions of altered consciousness scientifically. Their seminal publication "coma" is still considered a classic in todays advanced times.

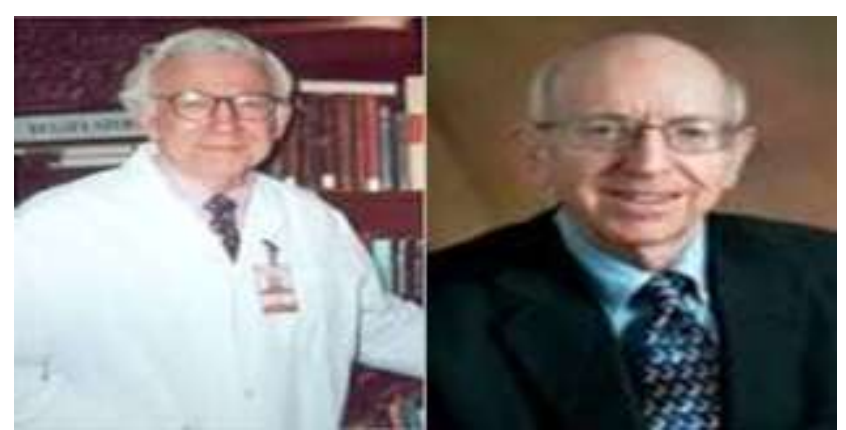

Fig. 3-4: Alfred Plum and George Posner

Consciousness is thus defined as a state of full awareness of the self and one's relationship to the environment. It was considered to consist of 2 components

1. Arousal or the level of behavior and innate responsiveness, and

2. Content or the sum of functions mediated by the cerebral cortex (cognitive and effective)

Consciousness was hence the ability to stay awake (arousal) as well as the sum total of all the thoughts and activities planned while awake (content).

Hence altered states of consciousness can be defined as

1. Disorders of Arousal such as lethargy, stupor, coma and

2. Disordersof Content such as Confusion, Hallucinations, emotional changes etc.

An important differentiation is to be made between organic causes of altered consciousness and Psychogenic unresponsiveness. Here patients although apparently unconscious show some response to external stimuli. For example, an attempt to elicit the corneal reflex may cause a vigorous contraction of the orbicularis oculi. There will also be marked resistance to passive movement of the limbs without signs of any disease/ organ damage to justify the state of consciousness.

\section{Altered states of Consciousness Include Confusion}

Here the major defect is a lack of attention with disorientation to time place and person. The patient thinks 
less clearly and more slowly with memory difficulties as well. There is also a Misinterpretation of external stimuli. Drowsiness may alternate with hyper -excitability and irritability as well.

\section{Torpidity}

Obtunded sensorium or mental blunting

\section{Stupor}

A condition of deep sleep or behavioral unresponsiveness from which arousal is only possible through violent stimulus.

\section{Clouding of Consciousness}

Minimally impaired awareness or wakefulness, which may alternate with hyper excitability.

\section{Delirium}

A disturbance of consciousness with impaired ability to focus, sustain or shift attention. It also involves a disturbance of cognition or Perception marked by disorientation, fear, irritability, and misperception of sensory stimuli. The patient is out of true contact with environment and other people

\section{Common Causes}

Toxins, metabolic disorders, partial complex seizures, head trauma, acute febrile systemic illnesses

\section{Coma}

A state of severely impaired consciousness from which the patient cannot be aroused even with vigorous stimuli.

\section{Locked in Syndrome}

A situation where the brain is de-efferented, by which response is impossible, but perception is intact.

\section{Dementia}

An enduring and progressive decline in mental prowess owing to an organic pathological process. Arousal is usually spared. There is a marked impairment of executive function localized to the cortex.

\section{Akinetic Mutism}

State of silent, yet alert appearing immobility ( anterior Basal forebrain and hypothalamus lesions)

\footnotetext{
Abulia

This implies a "lack of will" or slow response (if at all) to verbal stimuli, and inability to initiate activity. Cognition is unaffected. (localization is to bilateral frontal lobe involvement)
}

\section{Hypersomnia}

A state of excessive sleep from which the subject is readily arousable, but into which he relapses into readily.(hypothalamus is generally involved)

\section{Minimally concious state}

A condition of severely impaired consciousness in which minimal but definite behavioral evidence of self or environmental evidence is demonstrated. It is generally considered a transitional state during recovery or worsening.

\section{Persistent Vegetative State}

This denotes the recovery of the crude arousal cycle. Visceral regulation persists but content is absent. The term is used if the state persists for more than 30 days. Also known as Coma Vigil, and Apallic state.

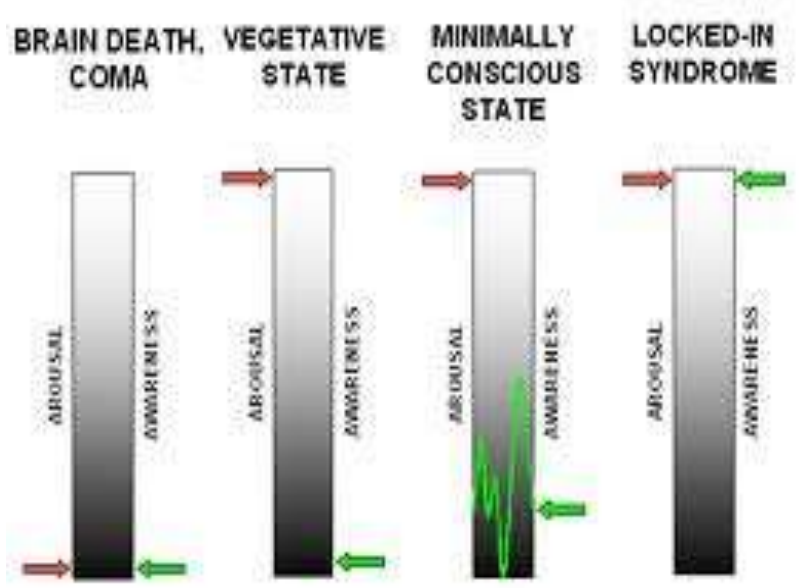

Fig. 5: A graphic comparison between the different states of altered conciousness

\section{Breathing Patterns in Patients of Altered Consciousness}

Cheyne-Stokes Pattern: This indicates diffuse hemispherical damage and localizes the lesion to the diencepalon.

Persistant Neurogenic Hyperventilation: This denotes a persistant hyperventilation without other more common cauases such as hyperthermia, acidosis, or hypoxia. It denotes a dysfunction at the level of the rostral midbrain.

\section{Apneusis}

This implies a mid to caudal pons localization with periodic peaks and troughs in the breathing rhythm without regularity or predictability of amplitude.

\section{Ataxic or Bitot's Breathing}

This indicates a lower medullary damage and is a chaotic mix of breathing patterns without gainful oxygenation. It is often considered a pre-terminal event. 


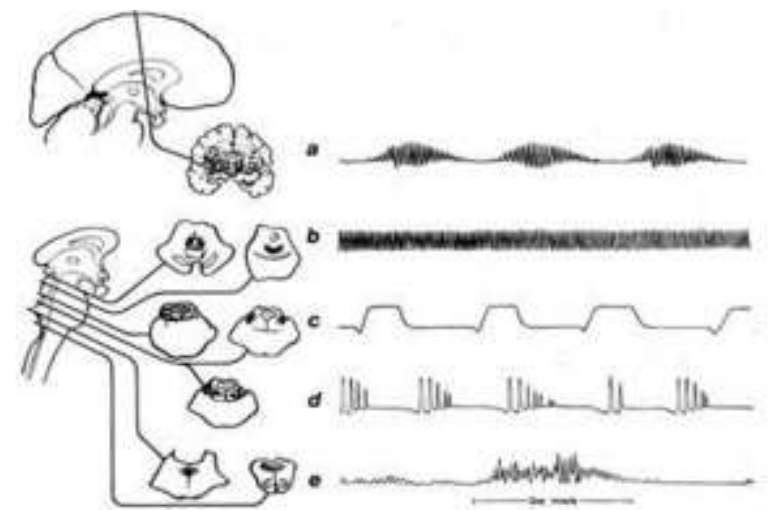

Fig. 6: Abnormal respiratory patterns associate with pathologic lesions (shaded areas) at various levels of the brain. Tracings by chest-abdomen pneumograph, inspiration reads up. a; cheyne-strokes respiration. b; Central neurogenic hyperventilation. c; Apneusis. d; Cluster breathing. e; Ataxic breathing.

\section{Signs of Lateralization}

Probably the most important signs required to picked up by any resident of nurse in the neurosurgical ICU or emergency are signs of lateralization which imply imminent herniation of either a part or the entire brain in the direction of the force vector it is subjected to due to a variety of pathologies present.

\section{These Include}

1. Unequal Pupils: These are by far the most significant and are a sign of impending Uncal Herniation

2. Gaze preference

3. Unilateral hypertonia

4. Asymmetrical deep tendon reflexes exaggerated

5. Unilateral Babinski reflex

6. Partial seizures

Causes of altered Consciousness

\section{Structural Causes}

1. Hematomas

2. Infarcts

3. Tumors

4. Abscess

5. Contusions

6. Gliomatosis

7. Hydrocephalus

8. ADEM

9. Leukoencephalopathy

\section{Metabolic Causes}

1. Dyselectrolytemias

2. DM

3. Liver disease

4. Renal disease

5. Endocrinopathies

6. Poisoning

7. Drug abuse/overdose

8. Seizures

9. Psychiatric unresponsiveness
Common causes encountered in neurocritical care include

\section{Metabolic Coma}

These Include

1. Diabetic coma: which maybe hyperosmolar non-ketotic or hypoglycemic in nature

2. Renal coma: which includes hyperuricemia as well as azotemic states. Of special mention is Dialysis Dysequilibrium syndrome where a complete loss of electrolytes and solutes leads to neuron swelling and encephalopathy.

3. Hepatic coma: well known as detectable in liver cell failure where bilirubin deposition in the basal nuclei leads to extrapyramidal states.

4. Thyroid coma: due to a complete fall of the BMR secondary to thyroid burnout or suppression (Myxedemic Coma)

5. Adrenal coma: secondary to insufficiency of cortisol and mineralocorticoid production in the face of rising demand.

6. Dyselectrolytemia: these include HypoNatremia, Hypernatremia as well as associated conditions such as:

\section{Siadh}

1. ADH release is related to the threshold of the thirst response and there is a lower threshold for thirst in patients with SIADH.

2. There is also loss of control of ADH release and plasma

3. SIADH is often a self-limiting disease after brain injury and treatment should only be initiated if the patient is symptomatic, the serum sodium is significantly low or falling rapidly.

\section{Cerebral Salt Wasting}

1. CSWS is characterized by renal loss of sodium resulting in polyuria, natriuresis, hyponatraemia, and hypovolaemia occurring as a result of a centrally mediated process.

2. It is predominantly associated with SAH and TBI but has also been described after brain tumour surgery, ischaemic stroke, and TB meningitis.

3. It usually occurs in the first week after brain injury and resolves spontaneously within 2-4weeks.

4. The primary treatment of CSWS is volume and sodium resuscitation.

5. CSWS may be refractory to standard therapy and fludrocortisone $(0.1-0.4 \mathrm{mg}$ daily) may limit the sodium loss by increasing sodium reabsorption.

\section{Diabetes Insipidus}

1. DI results from a failure of $\mathrm{ADH}$ release from the hypothalamic-pituitary axis.

2. The ability to concentrate urine is impaired resulting in the production of large volumes of dilute urine.

3. This inappropriate loss of water leads to an increase in serum sodium and osmolality and a state of clinical dehydration.

4. Management: fluid intake as per thirst in conscious patients and like for like replacement in comatose patients

5. Refractory cases require desmopressin 


\section{Intracranial Lesions causing raise in ICP and herniation.}

Intra Cranial Pressure is the pressure present inside the Calvarium or brain box. It is described as a function of the fixed volume of the space occupied by three components Brain, Blood and CSF (Monroe-Kelly Doctrine)

Total Volume $=$ Vol. of Brain + Vol. of Blood + Vol. of CSF

ICP is also functionally important is it opposes the perfusion of the brain as explained in the equation:

$\mathbf{C P P}=\mathbf{M A P}-\mathbf{I C P}$

Where the Cerebral Perfusion Pressure (CPP) is a function of the tussle of two opposing forces, Mean Arterial Pressure (MAP) that attempts to perfuse the brain parenchyma and the ICP that offers resistance to this positive pressure.

Hence it is clear that the greater the ICP, the lower the Perfusion Pressure and the worse is the resultant Hypoxia.

To compensate for the ICP rise, the brain attempts to auto-regulate the blood flow to meet perfusion demand at the same time to keep ICP within a range that permits brain function. The Auto regulation works with Chemical, Neural and Vascular Smooth Muscle acting in tandem, only within a specific range of pressures $(50-150 \mathrm{mmHg})$, beyond which a linear relationship is followed as shown below.

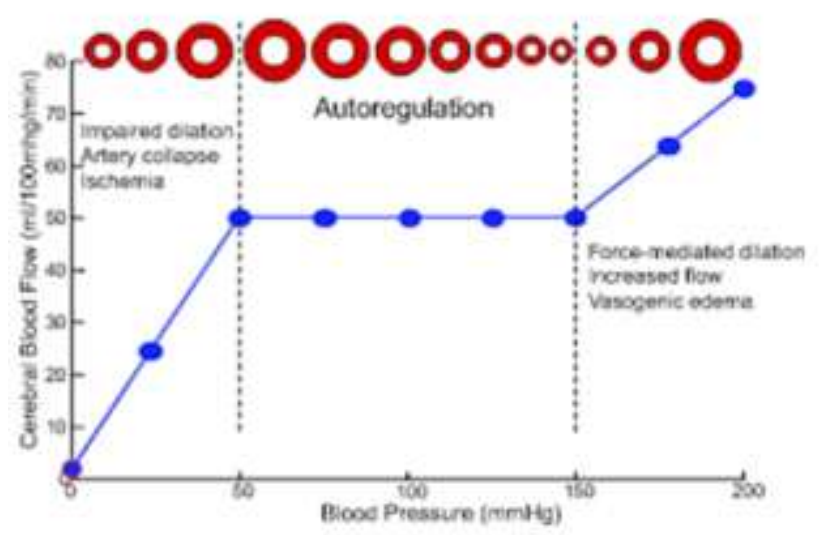

Fig. 7:

Persistent high ICP leads to Herniation of the semi solid Brain Matter through the various Foramina of the skull base, or through compartments in the cranial compartment in the direction of the Net Force Vector (NFV)

Increased ICP can be symmetrical, diffuse or localized.

\section{CVAs and vascular insufficiency}

Cerebrovascular disease is a frequent cause of coma. The cause ranges from Impairment of perfusion of the RAS due to block of the feeding vessels, hypotension to brainstem herniation (parenchymal haemorrhage, swelling from infarct, or more rarely, extensive brainstem infarction)
Decompressive craniectomies generally assist in reducing ICP due to cerebral edema theraby improving conciousness.

Hypotension is defined cerebrally as The critical blood flow in humans required to maintain effective cerebral activity is about $70 \mathrm{ml} / 100 \mathrm{~g} / \mathrm{min}$ in grey matter and $50 \mathrm{ml} / 100 \mathrm{~g} / \mathrm{min}$. Any fall below this leads rapidly to cerebral insufficiency. Hypotension leads to watershed hypoxia and can if persistant lead to infarcts. Suc scenarios are usually associated with excessive blood loss, cardiac conditions or shock which need to be assessed and managed in tandem.

\section{Head Trauma}

The leading cause of death below the age of 45 , head injury accounts for $1 / 2$ of all trauma deaths and is a major cause of patients presenting with coma. Alcohol on the breath provides a direct clue to a cause of coma, evidence of head injury need not necessarily imply that this is the cause.Epileptic seizure, may have resulted in a subsequent head injury.

Damage can be diffuse or focal, Different intensity injuries occur in different scenarios with Different force vectors. Each of the brains subjected to trauma and secondary injury have Different potentials for sustaining injury, raised ICP and hypoxia.

1. Diffuse axonal injury is now seen as the major consequence of head injury and associated coma.

2. Mild degrees of axonal injury also occur with concussion and brief loss of consciousness, which may recover spontaneously with differing levels of long term consequences to cognition, balance, memory and speech.

3. Subdural and extradural haematomata may cause impairment of consciousness following apparent recovery are important to diagnose, as they are readily treatable surgically

Secondary damage can occur from parenchymal haemorrhage, brain oedema, and vascular dilatation, all of which will lead to $\uparrow \mathrm{ICP} \rightarrow \downarrow$ perfusion pressure, which can be accentuated by systemic hypoxia and blood loss. The major long term morbidity and loss of man-hours of productivity occur due to the untreated secondary injury which when allowed to progress leads to devastating brain damage out of proportion sometimes to the original primary insult.

\section{Subarachnoid Haemorrhage}

Loss of consciousness is common with SAH. Only about $1 / 2$ of patients recover from the initial effects of the haemorrhage. SAH can be due to aneurysmal bleeds or a consequence of trauma. Both imply grevious brain damage. Subsequent sequelae include hyponatremia (SIADH or CSW) and vasospasm which further worsen the coma status of the patient from which recovery is often difficult, especially after the onset of infarcts. 


\section{DRUGS and POISONING}

Poisoning, drug abuse, and alcohol intoxication accounting for up to $30 \%$ of those presenting through accident and emergency departments, $80 \%$ of whom require only simple observation in their management.

The commonest form of intoxication is Alcoholism which is apparent from the history, flushed face, rapid pulse, and low blood pressure. The smell of alcohol on the breath. Intoxicated are at increased risk of hypothermia and of head injury can be the cause of coma. At low plasma concentrations of alcohol, mental changes, at higher levels, coma ensues, $>350 \mathrm{mg} / \mathrm{dl}$ may prove fatal.

Often Suicide is the aim of drug intoxication. The most commonly drugs in suicide attempts are Benzodiazepines, Paracetamol, antidepressants, and Narcotic overdoses (heroin)

\section{Seizures}

A Common cause of coma, with a period of unconsciousness following a single generalized seizure commonly lasting between 30 and 60 minutes. Following status epilepticus, there may be a prolonged period of coma. History, trauma to the tongue or inside of the mouth. Seizures secondary to metabolic disturbances may have a longer period of coma. Apart from trauma, the commonest cause of seizures include missed medication dosages and metabolic causes.

A fortunately rare cause which is getting rarer due to prompt management and early detection is Ecclampsia. This is seen in the second half of pregnancy and represents a failure of autoregulation, with raised blood pressure. Neuropathologically there are ring haemorrhages around occluded small vessels with fibrinoid deposits. Management stratgies include control of convulsions and raised blood pressure. Parental magnesium is commonly employed, which may give rise to hypermagnesaemia.

\section{Other Neurological Diseases}

These may include

1. PMLE

2. severe end-stage multiple sclerosis.

3. Prion disease may lead to coma over a short period of 6-8 weeks, but this is following a progressive course of widespread neurological disturbance

\section{Prognosis}

Management of COMA involves neurocritical care to ensure homeostasis along with appropriate management of the cause.

In general, coma carries a serious prognosis. Prognosis is dependent to a large extent on the underlying cause. Coma due to depressant drugs carries an excellent prognosis provided that resuscitative and supportive measures are available and no anoxia has been sustained. Metabolic causes, apart from anoxia, carry a better prognosis than structural lesions and head injury
Length of coma and increasing age are of poor prognostic significance. Absence of Brainstem reflexes early in the coma are an important predictor of outcome. The chronic vegetative state usually carries a uniformly poor prognosis, although a partial return of cognition, or even restoration to partial independence, has been reported very rarely.

Although unassociated with coma, the 'locked-in' syndrome also carries a poor prognosis, with only rare recoveries reported

\section{Conflict of Interest: None.}

\section{References}

1. Kaplan P, (2000) Prediction of poor outcome in anoxicischaemic coma: afterward. J Clin Neurophysiol 17:502.

2. Hodgson HJF, (1974) Coma after cardiac arrest. Brain 97:36172.

3. Willoughby JO, Leach BG, Relation of neurological findings after cardiac arrest to outcome. BMJ 1974;3(5929):437-9.

4. Snyder BD, Ramierz-Lassepas M, Lippert D, Neurologic status and prognosis after cardiopulmonary arrest; a retrospective study. Neurol 1977;27:807-811.

5. Jorgensen EO, Malchow-Moller, (1981) Natural history of global and critical brain ischaemia. Resuscitation 9:133-191.

6. Levy DE, Bates D,Caronna JJ. Prognosis in non-traumatic coma. Ann Intern Med 1981;94:293-301.

7. Levy DE, Caronna JA, Singer BH. Predicting outcome from hypoxic-ischaemic coma. JAMA 1985;253:1420-26.

8. Longstreth WT, Diehr P, Inuit S, Prediction of awakening after out of hospital cardiac arrest. N Engl J Med 1983;308:137882 .

9. Edgren E, Hedstren DU, Nordin M, Prediction of outcome after cardiac arrest. Crit Care Med 1987;15:820-25.

10. Zandbergen EGJ, de Haan RJ, Koelman JHTM, Prediction of poor outcome in anoxic-ischaemic coma. J Clin Neurophysiol 2000;17:498-501.

11. Pananicolaou AC, Loring EW, Eisdenberg HM, Prognostically important EEG coma patterns in diffuse anoxic and traumatic encephalopathies in adults. Neurosurg 1986;18:173-5.

12. Walser H, Murat E, Janzer R. Somato sensory evoked potentials in comatose patients: correlation with outcome of neurological findings. J Neurol 1986;233:34-40.

13. Roine RO, Somer H, Kaste M, Neurological outcome after out of hospital cardiac arrest: prediction by cerebrospinal fluid enzyme analysis. Arch Neurol 1989;46:823-43.

14. Jaggi JL, Obrist WD, Genneralli TA, Relationship of early cerebral blood flow and metabolism to outcome in acute head injury. J Neurosurg 1990;72:1761-82.

15. Shewmom DA, DeGiogio CM. Early prognosis in anoxic coma: reliability and rationale. Neurol Clin 1989;7:823-43.

16. The Multi-Society Task Force on PVS. Medical aspects of the persistent vegetative state. N Engl J Med 1994;330:1499-1508.

17. The Multi-Society Task Force on PVS (1994) Medical aspects of the persistent vegetative state. N Engl J Med 330:1572-9.

18. Working Group of the Royal College of Physicians. The permanent vegetative state. J R Coll Physicians Lond 1996;30:119-21.

How to cite this article: Ganapathy S, Gopal S, Ramakrishna TV. Coma and Conciousness. India J Neurosci 2019;5(2):426. 